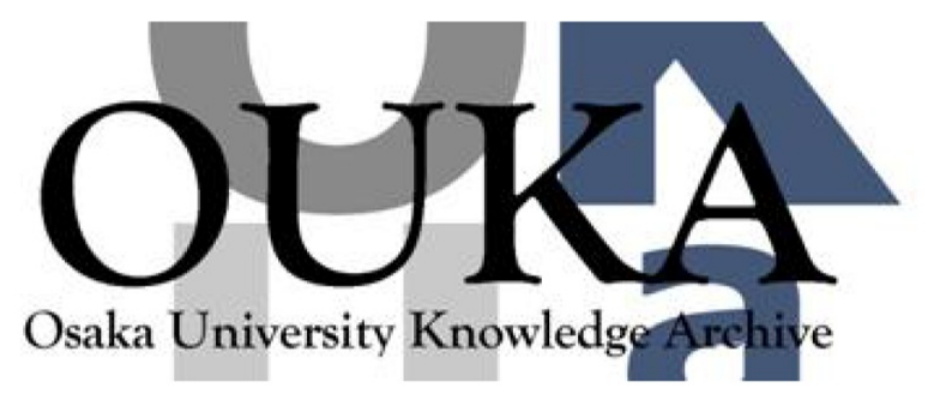

\begin{tabular}{|c|l|}
\hline Title & $\begin{array}{l}\text { Collusion through mediated communication in } \\
\text { repeated games with imperfect private } \\
\text { monitoring }\end{array}$ \\
\hline Author(s) & Aoyagi, Masaki \\
\hline Citation & Economic Theory. 25(2) p. 455-p. 475 \\
\hline Issue Date & $2005-02$ \\
\hline oaire:version & AM \\
\hline URL & https://hdl. handle.net/11094/3155 \\
\hline rights & \\
\hline Note & \\
\hline
\end{tabular}

Osaka University Knowledge Archive : OUKA

https://ir. Library. osaka-u. ac. jp/

Osaka University 


\title{
Collusion through Mediated Communication in Repeated Games with Imperfect Private Monitoring $\dagger$
}

\author{
MASAKi AOYAGI $\ddagger$
}

July 30, 1999

Revised: October 2, 2001

$\dagger$ I am very grateful to Mark Armstrong, V. Bhaskar, Michihiro Kandori and two referees of this journal for helpful comments. Part of this research was conducted while I was visiting the University College London. Their hospitality is gratefully acknowledged.

†ISER, Osaka University, 6-1 Mihogaoka, Ibaraki, Osaka 567-0047, Japan. E-mail address: aoyagi@iser.osaka-u.ac.jp. 


\section{Abstract}

This paper studies repeated games with imperfect private monitoring when there exists a third-party mediator who coordinates play by giving non-binding instructions to players on which action to take and by collecting their private information. The paper presents a Nash-threat folk theorem for a communication equilibrium based on such mediation when monitoring is jointly $\epsilon$-perfect in the sense that every player is almost perfectly monitored collectively by other players.

Journal of Economic Literature Classification Numbers: C72, D82.

Key Words: repeated games, private monitoring, joint monitoring, communication, mediation. 


\section{Introduction}

Repeated games with imperfect private monitoring, where players' actions in each period give rise to noisy private signals instead of a publicly observable signal, provide an accurate description of many competitive situations involving moral hazard. A frequently cited example is the repeated Bertrand oligopoly where the competing firms may offer secret price discounts to their customers (Stigler (1964)). When one firm offers such a discount, other firms will experience a stochastic reduction in their demand level. There is, however, no public signal which indicates this change. It is now well recognized that the essential difficulty associated with private monitoring is the lack of a coordination device on which play can be conditioned. To see this, note that the standard grim-trigger strategy in repeated games with (imperfect) public monitoring starts punishment at a particular realization of the public signal. In quantity setting oligopoly with publicly observed price, for example, price below a certain threshold triggers punishment whether that is caused by cheating of a cartel member or a poor market condition. It can be seen that without a public signal, coordinated reversion to punishment at exactly the same timing would be impossible.

When faced with the coordination problem, however, it is natural to expect that players attempt various forms of communication with one another. In fact, empirical findings suggest that communication is a key component of many collusion schemes. Among others, Scherer (1980) reports a number of anti-trust cases against "trade associations," a gathering of representatives from rival companies for the exchange of information. ${ }^{1}$ The principal purpose of this paper is to analyze player communication in repeated games in order to understand collusion and cooperation in the absence of public signals.

Matsushima (1991), Compte (1994, 1998), and Kandori and Matsushima (1998) are the first to analyze player communication in repeated games with private monitoring. These models assume that communication is public in the sense that players report their private signals publicly and simultaneously. While public communication is a simple and natural form of communication, there is no reason to believe that parties to a complex collusion scheme do not employ a more sophisticated communication method. For example, Doyle and Snyder (1999) study the interaction of the "Big 4" U.S. automakers during 1965-

\footnotetext{
${ }^{1}$ Genesove and Mullin (2001) give a detailed documentation of such meetings in the U.S. sugar industry between 1927 and 1936.
} 
95 through a leading industry trade journal: The journal privately contacts each automaker to elicit their monthly production plans up to six months ahead of actual production. Upon publication, it privately contacts each producer again to obtain a revised plan for publication in the next issue. The journal repeats this process and publishes a series of revisions up to the month in question. This episode suggests not only the potential complexity of a communication process but also the existence of a mediator who engages in secret communication with firms.

In this light, this paper assumes the most general form of communication and examines its consequences. Formally, the paper analyzes mediated communication as proposed by Myerson (1986) and Forges (1986), where a third-party mediator coordinates play. Specifically, the mediator gives secret instructions to players on which action to play and collects their private signals to determine future instructions. Following Forges (1986), we refer to an equilibrium of such a mediation game as a communication equilibrium. The critical difference between public and mediated communication is as follows: In the models of public communication, players' (possibly mixed) actions in each period are conditioned only on the public reports of their signals. Under mediated communication, in contrast, their actions are conditioned on secret instructions from the mediator and hence may be correlated. In this sense, the relationship between equilibrium based on public communication and that based on mediated communication parallels the relationship between Nash and correlated equilibrium in normal-form games.

We do not specify the nature of the mediator in our model. In some applications, it would be natural to suppose that the role of mediation is performed by an individual or organization such as the trade journal mentioned above. When taking this interpretation, however, it should be noted that we have assumed away the incentive problem faced by the mediator himself. In any event, the mediator's task is to receive reports from the players as inputs and give secret instuctions to them as outputs (with some randomization). In particular, it should be noted that the mediator is not required to observe any information first-hand. The scheme is set up so that he will obtain all the relevant information from the players in an incentive compatible manner.

Along with mediated communication, this paper focuses on the possibility of joint monitoring under private monitoring. Under public monitoring, every player is monitored 
by all other players through a common signal. Under private monitoring, on the other hand, the relationship between a player and the identity and nature of his monitors takes various forms. Ben-Porath and Kahnemann (1996) analyze a model of repeated games in which each player's action is perfectly monitored by some subset of his opponents. Joint monitoring in their context is hence summarized by the identity of the monitors of each player. In general, however, if a player has two or more monitors, then their monitoring technologies may be different from each other: First, different monitors may observe different aspects of his activity. For example, if the player is a middle manager of any company, then his job would have many different aspects which are observed separately by disjoint sets of people: His leadership qualities are monitored by his subordinates, creative thinking by his boss, dedication to customer service by clients, etc. Comprehensive assessment of his performance requires their joint inputs. ${ }^{2}$ Second, when monitoring is imperfect, the monitors' (noisy) signals may vary in accuracy, correlation with one another, and so on, even if they are generated by a single action of the monitored player. In both cases, monitoring would be most accurate when the monitors' signals are jointly evaluated.

This paper presents a Nash folk theorem for communication equilibrium when monitoring is jointly $\epsilon$-perfect in the sense that the collective assessment of the monitors' signals reveals the player's action almost perfectly.

From a technical point of view, analysis of repeated games with communication under almost perfect monitoring is important for two reasons: First, it will furnish robustness to the theory of repeated games based on perfect monitoring against a slight perturbation in the monitoring technology. As will be seen, the monitoring structure assumed in this paper subsumes many natural and interesting classes of near perfect monitoring. Second, games with almost perfect monitoring are the subject of the analysis which does not assume player communication: Bhaskar and van Damme (1997), Ely and Valimaki (2000), Mailath and Morris (1999), Obara (2000), Piccione (1999), Sekiguchi (1997, 1998) all discuss the possibility of an efficient equilibrium under near perfect monitoring. Although one might expect a comparable conclusion with communication, the existing analysis is not readily applicable. As expalined below, mediated communication also restores and generalizes the

\footnotetext{
${ }^{2} \mathrm{An}$ appraisal system based on this class of joint monitoring is known as "360-degree feedback" in organization theory, and is gaining popularity in actual practice. (See, for example, Fletcher (1997).)
} 
critical observation by Bhaskar and Van Damme (1997) in a game without communication.

In what follows, we illustrate how mediated communication is used to sustain cooperation in our model. The general difficulty associated with public communication as modeled by Kandori and Matsushima (1998) and Compte $(1994,1998)$ is the provision of a proper incentive for truth-telling. It should be noted that truth-telling in public communication requires each player's continuation payoff to be either independent of his report, or dependent also on reports from the other players. Otherwise, a player will always report the signal that maximizes his continuation payoff regardless of his true signal. Unlike public communication, mediated communication allows imperfect correlation of players' actions through secret instructions given to them by the mediator. A collusion scheme can exploit this uncertainty. To be concrete, suppose that players want to sustain cooperation in the repeated prisoners' dilemma. Consider first the grim-trigger scheme in which the mediator instructs each player to play " $C$ " if no "bad" signal has been reported in the past, and " $D$ " otherwise. While this is a natural extension of the standard grim-trigger strategy under public monitoring, it does not work under private monitoring: If player $i$ expects the other player $j$ to play $C$ as instructed, then $i$ does not want to report a bad signal truthfully since he knows that every bad signal is just an error, and that truthful revelation would end cooperation prematurely. If player $j$ knows that $i$ would never report a bad signal, however, then $j$ has no incentive to choose $C$ in the first place. The problem is that the incentive for truth-telling cannot be ensured when a player is certain about the other player's action. Consider now a variation of the above grim-trigger scheme where the mediator's instruction is " $D$ " with small but positive probability during the cooperation phase. Reversion to the punishment phase takes place if and only if player $i$ reports a bad signal when the instruction to player $j$ was " $C$," or $i$ reports a "good" signal when the instruction to $j$ was " $D$ " $(i \neq j)$. Each player now finds it optimal to report his signal truthfully: If the signal is bad, then it is likely that the other player has been instructed to play $D$.

It is instructive to compare the above logic to that used in Ben-Porath and Kahnemann (1996). In their basic model, each player $i$ is perfectly monitored by two or more players. The monitors are induced to tell the truth through the punishment inflicted on them in 
the event that their reports about $i$ are incompatible with one another. ${ }^{3}$ Although it is tempting to apply this logic to the imperfect monitoring environment considered in the present paper, a straight extension encounters the same kind of difficulty as described above. If player $i$ is expected to play action $C$ with probability one, then any monitor who receives a bad signal about $i$ will not report it truthfully because he knows that it is an error in his observation and expects other monitors to report good signals about $i$ with high probability. Anticipating this, player $i$ will deviate to $D$. As will be seen, this problem is avoided if the signals reported by $i$ 's monitors are matched against the instruction given to $i$, rather than against each other.

The comparison of results available under mediated and public communication is as follows: First, in two-person games, the folk theorems of Compte (1998) and Kandori and Matsushima $(1994,1998)$ both place certain restrictions on the stage-game payoffs along with the informational condition known as conditional independence. ${ }^{4}$ While these results do not require near perfect monitoring, Compte (1998) demonstrates that the set of (perfect public) equilibrium payoffs is in general strictly smaller than the set of feasible payoffs above the one-shot Nash equilibrium point (regardless of the level of monitoring and the discount factor). No restriction on stage-payoffs is necessary for our result. Second, in games with three or more players, the folk theorems of Compte (1994) and Kandori and Matsushima (1998) require that the probability distribution of private signals satisfy the "distinguishability conditions," which enable players to statistically identify a potential deviator. While these conditions do not imply near perfect monitoring and are generically satisfied if the number of signals is sufficiently large (compared to the number of actions) and if monitoring is "global" in the sense that every player is monitored by every other player, they are not necessarily implied by jointly $\epsilon$-perfect monitoring as assumed in this paper. For example, they fail to hold if some player is monitored by only one other player.

The logic behind the use of mediated communication is most closely related to the observation by Bhaskar and van Damme (1997), who note that for a player's private signal to be a useful indicator of the opponent's action, the opponent must be randomizing his actions. The technical aspect of the analysis in this paper bears some resemblance to that

\footnotetext{
${ }^{3}$ For example, one monitor reports a good signal while the other reports a bad signal.

${ }^{4}$ Conditional independence requires the players' signals to be independent of each other conditional on their action profile.
} 
of Aoyagi (2000), which studies public communication under imperfect private monitoring when the private signals are correlated. While there may be no exogenous correlation between private signals in the current framework, correlation is endogenously generated between the actions and the players' private signals through the mediator's instructions. While the discussion of this paper is totally embedded in the repeated game framework, the importance of mediated communication is noted in other contexts as well. In particular, Mitusch and Strausz (2000) examine the role of a mediator in a principal-agent problem and show that the random instructions by the mediator can be used to alleviate the commitment problem faced by the principal.

The organization of the paper is as follows. The next section gives a formal description of a communication game in an infinitely repeated game setting. Section 3 describes jointly $\epsilon$-perfect monitoring with some examples. The folk theorem is presented in Section 4 . Section 5 compares public and mediated communication.

\section{Communication Games}

Our formulation of a communication game is essentially identical to that of Myerson (1986) and Forges (1986). It is, however, specifically adapted to the infinitely repeated environment studied in this paper.

The set $I=\{1, \ldots, n\}$ of $n$ players and a mediator interact over an infinite number of periods. Denote by $A_{i}$ the finite set of player $i$ 's actions and by $A=\prod_{i \in I} A_{i}$ the set of action profiles. The set of player $i$ 's mixed actions is denoted $\Delta A_{i} \cdot{ }^{5}$ Action profile $a \in A$ stochastically determines each player $i$ 's private signal $s_{i}$, which is an element of the finite set $S_{i}$. The probability of signal profile $s=\left(s_{i}\right)_{i \in I} \in S$ under action profile $a \in A$ is denoted by $P(s \mid a) .{ }^{6}$ Player $i$ 's stage-payoff function is given by $g_{i}: A \rightarrow \mathbf{R}$, and the set of feasible payoff vectors is denoted by

$$
V=\operatorname{co}\left\{\left(g_{i}(a)\right)_{i \in I}: a \in A\right\}
$$

where co denotes the convex hull. A communication game is a repeated game in which the mediator gives secret instructions to players on which action to take before each stage-game,

\footnotetext{
${ }^{5}$ For any set $A, \Delta A$ denotes the set of probability distributions over $A$ endowed with an appropriate $\sigma$-algebra.

${ }^{6}$ Throughout, $\tilde{s}$ denotes a random variable and $s$ its realization. Where no confusion arises, the event $\tilde{s}=s$ is expressed simply as $s$.
} 
and then collects reports on their private signals at the end of each period. Specifically, it is characterized by the set $Q_{i}$ of possible instructions to player $i$, and the set $R_{i}$ of his reports $(i \in I) .{ }^{7}$ Write $Q=\prod_{i \in I} Q_{i}$ and $R=\prod_{i \in I} R_{i}$. In general, no restrictions will be placed on the nature of the instruction: It may be a summary of information on the history of play that the mediator has collected, or a suggestion as to which action should be taken based on such information.

Player $i$ 's action rule $\lambda_{i}: Q_{i} \rightarrow \Delta A_{i}$ chooses the mixed action as a function of the current instruction from the mediator, and his reporting rule $\mu_{i}: Q_{i} \times A_{i} \times S_{i} \rightarrow \Delta R_{i}$ specifies the mixed report as a function of the instruction as well as his own action and signal. Denote by $C_{i}$ the set of pairs $\left(\lambda_{i}, \mu_{i}\right)$ of player $i$ 's action and reporting rules.

Player $i$ 's communication history after period $t$ is a sequence of the mediator's instructions to him and his own reports in periods $1, \ldots, t$. On the other hand, player $i$ 's private history after period $t$ is a sequence of his own actions and private signals in periods $1, \ldots, t$.

Player $i$ 's (behavioral) strategy $\sigma_{i}: \bigcup_{t=0}^{\infty}\left\{\left(Q_{i} \times R_{i}\right)^{t} \times\left(A_{i} \times S_{i}\right)^{t}\right\} \rightarrow \Delta C_{i}$ determines the probability distribution over pairs of action and reporting rules $\left(\lambda_{i}, \mu_{i}\right)$ as a function of his communication and private histories. Player $i$ 's strategy $\sigma_{i}$ is simple if it is a function of his communication histories alone.

On the other hand, the mediator's choice of instructions is captured by the (behavioral) mediation strategy $\tau: \bigcup_{t=0}^{\infty}(Q \times R)^{t} \rightarrow \Delta Q$, which determines a (mixed) instruction profile to all players as a function of their communication histories.

The players have a common discount factor $\delta \in(0,1)$, and player $i$ 's (average) discounted repeated game payoff $V_{i}(\sigma, \tau, \delta)$ (normalized by $(1-\delta)$ ) under the strategy profile $(\sigma, \tau)$ is defined in the usual manner. A communication equilibrium is a strategy profile $(\sigma, \tau)$ such that $V_{i}(\sigma, \tau, \delta) \geq V_{i}\left(\sigma_{i}^{\prime}, \sigma_{-i}, \tau, \delta\right)$ for each $\sigma_{i}^{\prime}$ and $i \in I .{ }^{8}$ A communication equilibrium $(\sigma, \tau)$ is simple if each $\sigma_{i}(i \in I)$ is simple.

\footnotetext{
${ }^{7}$ If the set of possible instructions or reports depends on the history of play, $Q_{i}$ or $R_{i}$ can be redefined to be the union of all such sets.

${ }^{8}$ In Myerson (1986), a communication equilibrium is the mediation strategy $\tau$ itself if the profile $\sigma^{*}$ of honest and obedient strategies forms a Nash equilibrium under $\tau$. Although we will indeed consider such an equilibrium in Section 4, the present definition is convenient for the discussion in Section 5.
} 


\section{Jointly $\epsilon$-Perfect Monitoring}

Given $\epsilon \geq 0$, monitoring is jointly $\epsilon$-perfect if for every $i \in I$, there exists a partition $\Omega_{-i}=\left\{\Omega_{-i}\left(a_{i}\right)\right\}_{a_{i} \in A_{i}}$ of $S_{-i}$ such that

$$
P\left(\tilde{s}_{-i} \in \Omega_{-i}\left(a_{i}\right) \mid a_{i}, a_{-i}\right) \geq 1-\epsilon \text { for every }\left(a_{i}, a_{-i}\right) \in A \text {. }
$$

With jointly $\epsilon$-perfect monitoring, hence, player $i$ 's action $a_{i}$ gives rise to a signal profile in $\Omega_{-i}\left(a_{i}\right)$ with high probability. In other words, $i$ 's action is almost perfectly monitored by other players when their private signals are jointly evaluated. Note that this definition does not preclude the possibility that a player is monitored only by a proper subset of his opponents. ${ }^{9}$

A special case of the above definition is as follows: Monitoring is $\epsilon$-perfect if for every $i \in I$ and $j \neq i$, there exists a partition $\Omega_{j}=\left\{\Omega_{j}\left(a_{i}\right)\right\}_{a_{i} \in A_{i}}$ of $S_{j}$ such that

$$
P\left(\tilde{s}_{j} \in \Omega_{j}\left(a_{i}\right) \text { for every } j \neq i \mid a_{i}, a_{-i}\right) \geq 1-\epsilon \text { for every }\left(a_{i}, a_{-i}\right) \in A \text {. }
$$

With $\epsilon$-perfect monitoring, therefore, each player almost perfectly monitors the action profile of all other players. By letting $\Omega_{-i}\left(a_{i}\right)=\prod_{j \neq i} \Omega_{j}\left(a_{i}\right)$, it can be seen that $\epsilon$ perfection implies joint $\epsilon$-perfection. The two notions coincide in two-person games. $\epsilon-$ perfect monitoring is canonical if for each $j \in I, s_{j}=\left(s_{j}^{1}, \ldots, s_{j}^{j-1}, s_{j}^{j+1}, \ldots, s_{j}^{n}\right), s_{j}^{i} \in A_{i}$ $(i \neq i)$, and

$$
P\left(\tilde{s}_{j}^{i}=a_{i} \text { for every } j \neq i \mid a_{i}, a_{-i}\right) \geq 1-\epsilon \text { for every }\left(a_{i}, a_{-i}\right) \in A \text {. }
$$

In a canonical model, hence, the set of player $j$ 's signals is isomorphic to the set of action profiles of all other players.

Suppose that monitoring $P$ is jointly $\epsilon$-perfect with partitions $\left\{\Omega_{-i}\right\}_{i \in I}$. For each $i \in I$ and $s_{-i} \in S_{-i}$, define $a_{i}^{*}\left(s_{-i}\right)$ to be the unique action $a_{i} \in A_{i}$ of player $i$ such that $s_{-i} \in \Omega_{-i}\left(a_{i}\right)$. In other words, we have

$$
s_{-i} \in \Omega_{-i}\left(a_{i}^{*}\left(s_{-i}\right)\right) \text { for every } i \in I \text { and } s_{-i} \in S_{-i} \text {. }
$$

\footnotetext{
${ }^{9}$ For example, if player $j$ does not monitor player $i$, then choose the partition $\Omega_{-i}$ of $S_{-i}$ so that each $\Omega_{-i}\left(a_{i}\right)$ is a cylinder set with base $S_{j}$. This way, $j$ 's signal will be ignored.
} 
Jointly $\epsilon$-perfect monitoring $\left(P,\left\{\Omega_{-i}\right\}_{i \in I}\right)$ is consistent if for every $j \in I, s_{j}, r_{j} \in S_{j}$, $a_{j} \in A_{j}$, and $i \neq j$,

$$
\begin{aligned}
& \sum_{a_{-i-j}} \sum_{s_{-i-j}} P\left(s_{j}, s_{-i-j} \mid a_{i}=a_{i}^{*}\left(s_{j}, s_{-i-j}\right), a_{j}, a_{-i-j}\right) \\
& \geq \sum_{a_{-i-j}} \sum_{s_{-i-j}} P\left(s_{j}, s_{-i-j} \mid a_{i}=a_{i}^{*}\left(r_{j}, s_{-i-j}\right), a_{j}, a_{-i-j}\right) .
\end{aligned}
$$

Consistency is used to derive the optimality of truth-telling when the players report their signals to the mediator. While the direct interpretation of this condition is somewhat difficult, it permits some intuitive sufficient conditions as discussed below.

First, it can be verified that the standard class of $\epsilon$-monitoring (1) is consistent.

Proposition 1. For $\epsilon \geq 0$ small, if monitoring is $\epsilon$-perfect and canonical, then it is consistent.

Proof: See the Appendix.

Next, jointly $\epsilon$-perfect monitoring $\left(P,\left\{\Omega_{-i}\right\}_{i \in I}\right)$ is congruent if for each $i \in I$ and $s_{-i} \in S_{-i}$,

$$
P\left(s_{-i} \mid a_{i}=a_{i}^{*}\left(s_{-i}\right), a_{-i}\right)=\max _{a_{i} \in A_{i}} P\left(s_{-i} \mid a_{i}, a_{-i}\right) \quad \text { for any } a_{-i} \in A_{-i} .
$$

In other words, given any signal profile $s_{-i}, i$ 's action $a_{i}^{*}\left(s_{-i}\right)$ maximizes the probability of $s_{-i}$ regardless of the action profile of other players. Put differently, congruency asserts that the inference by $i$ 's monitors about his most likely action depends only on $i$ 's action and not on others'. ${ }^{10}$ It can be readily verified that congruency implies consistency, and that the two conditions coincide in two-person games. Congruency is easy to check in actual problems.

For illustration, suppose that in a two-person game, the set of actions is given by $A_{i}=\{C, D\}$ and the set of signals is given by $S_{i}=\{0,1,2\}(i=1,2)$. Assume that the

\footnotetext{
${ }^{10}$ In this sense, it would not be unreasonable to include congruency as part of the definition of $\epsilon$-jointly perfect monitoring.
} 
probability distribution of $\tilde{s}_{j}$ is as follows $(j \neq i)$ :

$$
\begin{aligned}
& a_{j} \quad a_{j} \quad a_{j} \\
& \begin{array}{lllllll}
C & D & C & D & C & D
\end{array}
\end{aligned}
$$

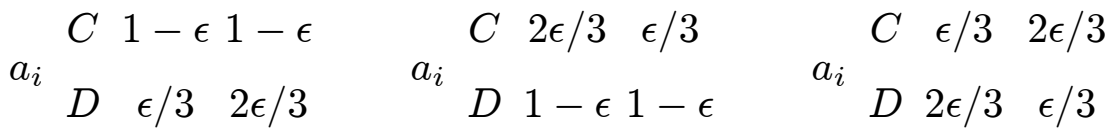

$$
\begin{aligned}
& P\left(\tilde{s}_{j}=0 \mid a\right) \quad P\left(\tilde{s}_{j}=1 \mid a\right) \quad P\left(\tilde{s}_{j}=2 \mid a\right)
\end{aligned}
$$

Figure 1

As seen, $\tilde{s}_{j}=0$ strongly indicates $a_{i}=C$, while $\tilde{s}_{j}=1$ strongly indicates $a_{i}=D$, both regardless of $j$ 's own action. Indeed, monitoring is $\epsilon$-perfect with $\Omega_{j}\left(a_{i}=C\right)=\{0,2\}$ and $\Omega_{j}\left(a_{i}=D\right)=\{1\}$. This distribution, however, violates congruency (and consistency) since $i$ 's action that maximizes the probability of $\tilde{s}_{j}=2$ changes with $j$ 's own action as

$$
\begin{aligned}
& P\left(\tilde{s}_{j}=2 \mid a_{i}=C, a_{j}=C\right)<P\left(\tilde{s}_{j}=2 \mid a_{i}=D, a_{j}=C\right), \quad \text { but } \\
& P\left(\tilde{s}_{j}=2 \mid a_{i}=C, a_{j}=D\right)>P\left(\tilde{s}_{j}=2 \mid a_{i}=D, a_{j}=D\right) .
\end{aligned}
$$

In the above example, monitoring fails to be congruent (or consistent) because of the existence of a signal which occurs with small probability under any action of player $i$. The following proposition shows that joint $\epsilon$-perfection implies congruency if each signal profile $s_{-i}$ occurs with probability $\geq \epsilon$ under some $a_{i}$.

Proposition 2. Suppose that monitoring is jointly $\epsilon$-perfect. If for every $i \in I, s_{-i} \in S_{-i}$ and $a_{-i} \in A_{-i}$,

$$
\max _{a_{i} \in A_{i}} P\left(s_{-i} \mid a_{i}, a_{-i}\right) \geq \epsilon
$$

then monitoring is congruent.

Proof: See the Appendix.

Since the above inequality holds automatically when there is no noise (i.e., $\epsilon=0)$, it immediately follows from Proposition 2 that monitoring is congruent whenever it is jointly 0 -perfect.

The following are some examples of jointly $\epsilon$-perfect monitoring.

EXAMPLE 1: Monitoring is locally $\epsilon$-perfect if for every $i \in I$, there exists a non-empty set $E_{i} \subset I \backslash\{i\}$ such that for each $j \in E_{i}$, there exists a partition $\Omega_{j}=\left\{\Omega_{j}\left(a_{i}\right)\right\}_{a_{i} \in A_{i}}$ such 
that

$$
\begin{aligned}
& P\left(\tilde{s}_{j} \in \Omega_{j}\left(a_{i}\right) \text { for every } j \in E_{i} \mid a_{i}, a_{-i}\right) \geq 1-\epsilon \text { for every }\left(a_{i}, a_{-i}\right) \in A, \text { and } \\
& P\left(s_{-E_{i}} \mid a\right)=P\left(s_{-E_{i}} \mid a_{i}^{\prime}, a_{-i}\right) \text { for every } s_{-E_{i}} \in S_{-E_{i}}, a \in A, \text { and } a_{i}^{\prime} \in A_{i} .
\end{aligned}
$$

Namely, each player $i$ is $\epsilon$-perfectly monitored by every player in some subset $E_{i}$, but no other player observes $i$ 's action. This would be the case, for example, in network economies where player $i$ is monitored by another player $j$ if and only if $i$ is linked to $j$. Ben-Porath and Kahneman (1997) analyze games with locally 0-perfect monitoring. ${ }^{11}$

In games with three or more players, the distinguishability conditions of Kandori and Matsushima (1998) are implied by (global) $\epsilon$-perfection (1) as long as $\epsilon>0$ is small enough. As seen below, however, they are not necessarily implied by joint $\epsilon$-perfection. To see this with local $\epsilon$-perfection, suppose that monitoring is locally $\epsilon$-perfect, and that there exists $i \in I$ such that $E_{i}=\{j\}$ for some $j \in I$. That is, player $j$ is the only monitor of player $i$, and no other player receives a signal about $i$ 's action. Given any signal profile $s_{-i-j} \in S_{-i-j}$ of players other than $i$ and $j$, it is straightforward to verify that $P\left(s_{-i-j} \mid a\right)=P\left(s_{-i-j} \mid a_{i}^{\prime}, a_{-i}\right)$ for every $a \in A$ and $a_{i}^{\prime} \in A_{i}$. This violates condition (A2) of Kandori-Matsushima (1998).

EXAMPLE 2: Suppose that a monopolist (player 0) sells his product to a large number of consumers (players $1, \ldots, n)$. The monopolist's effort (action) in the production process stochastically determines the product qualities that are privately observed by the consumers. Even if each consumer's signal is noisy, joint evaluation of all their signals reveals the monopolist's action with high accuracy by the law of large numbers (provided that those signals are independent across consumers conditional on the monopolist's action).

For concreteness, suppose that the monopolist has two actions high $(H)$ and low $(L)$, and that the quality of the good delivered to each consumer $i$ is either 0 or $1: A_{0}=\{L, H\}$ and $S_{i}=\{0,1\}(i=1, \ldots, n)$. The probability distribution $P$ is such that $s_{1}, \ldots, s_{n}$ are independent conditional on $a_{0}$ and for every $i=1, \ldots, n$,

$$
P\left(s_{i}=1 \mid a_{0}=H, a_{-0}\right)=P\left(s_{i}=0 \mid a_{0}=L, a_{-0}\right)=\gamma \quad \text { for any } a_{-0} \in A_{-0},
$$

\footnotetext{
${ }^{11}$ As pointed out in the Introduction, however, there exists a difference between the logic behind the construction of an equilibrium in this paper and that in Ben-Porath and Kahneman (1997).
} 
where $\gamma$ is a constant. We assume that $\gamma>1 / 2$ so that $s_{i}=1$ and $s_{i}=0$ represent high and low quality, respectively. Define

$$
\Omega_{-0}\left(a_{0}=H\right)=\left\{s_{-0} \in S_{-0}: \sum_{i=1}^{n} s_{i} \geq \frac{n}{2}\right\}, \quad \Omega_{-0}\left(a_{0}=L\right)=\left\{s_{-0} \in S_{-0}: \sum_{i=1}^{n} s_{i}<\frac{n}{2}\right\} .
$$

It directly follows from the law of large numbers that for any $\gamma>1 / 2$ and $\epsilon>0$, we can take $n$ large enough so that monitoring of the monopolist is jointly $\epsilon$-perfect. To see that it is also consistent, note that

$$
a_{0}^{*}\left(s_{-0}\right)= \begin{cases}H & \text { if } s_{-0} \in \Omega_{-0}(H) \\ L & \text { if } s_{-0} \in \Omega_{-0}(L)\end{cases}
$$

We have for any $s_{-0} \in S_{-0}$,

$$
P\left(s_{-0} \mid a_{0}=a_{0}^{*}\left(s_{-0}\right), a_{-0}\right) \geq P\left(s_{-0} \mid a_{0}, a_{-0}\right) \quad \text { for any }\left(a_{0}, a_{-0}\right) \in A \text {. }
$$

This shows that monitoring is congruent.

EXAmPle 3: Suppose that player $i$ has an $n_{i}$-dimensional $\left(n_{i} \geq 2\right)$ action, and the $k$ th dimension of his action is closely monitored by player $k$ in the set $E_{i} \subset I \backslash\{i\}$. Formally, let $a_{i}=\left(a_{i}^{1}, \ldots, a_{i}^{n_{i}}\right) \in A_{i}=\prod_{k=1}^{n_{i}} A_{i}^{k}$ and $S_{k}=A_{i}^{k}$ for each $k \in E_{i}$, and suppose that

$$
P\left(\tilde{s}_{k}=a_{i}^{k} \text { for every } k \in E_{i} \mid a_{i}, a_{-i}\right) \geq 1-\epsilon \text { for every }\left(a_{i}, a_{-i}\right) \in A
$$

For example, if player $i$ is a middle manager subject to the 360-degree feedback system mentioned in the Introduction, then $E_{i}$ is a collection of $i$ 's subordinates, peers, boss, clients, and so on. If $i$ is an assistant professor whose job is to teach and to do research, then $E_{i}$ would consist of his colleagues and students.

\section{Nash Folk Theorem}

In what follows, we fix a Nash equilibrium $m^{e} \in \prod_{i \in I} \Delta A_{i}$ of the stage-game and normalize the associated stage-payoff $g_{i}\left(m^{e}\right)$ to zero for every player.

Theorem 1. Take any $v^{*} \in V$ such that $v_{i}^{*}>0$ for every $i \in I$. For any $\xi>0$, there exists $\epsilon>0$ such that the following holds if monitoring is jointly $\epsilon$-perfect and consistent: 
For $\delta$ sufficiently close to one, there exists a communication equilibrium $\left(\sigma^{*}, \tau\right)$ with the payoff $v_{i}(\delta)=V_{i}\left(\sigma^{*}, \tau, \delta\right)$ satisfying $\left|v_{i}(\delta)-v_{i}^{*}\right|<\xi$ for every $i \in I$.

The proof of the theorem is based on the following construction. Suppose first that for some integer $T$, the entire repeated game is partitioned into $T$ separate "component games": Component game 1 consists of periods $1, T+1,2 T+1, \ldots$, component game 2 consists of periods $2, T+2,2 T+2, \ldots$, and so on. Actions taken in each period affect continuation play in the same component game only. ${ }^{12}$

The mediation strategy $\tau$ is the grim-trigger type in each component game: The game begins in the cooperation phase, and reverts to the punishment phase after some history. In any period during the cooperation phase, the mediator first chooses whether or not any player will be monitored in that period. He then randomly chooses an action profile for all players to play. If an action profile $a=\left(a_{i}\right)_{i \in I}$ is chosen, the instruction $a_{i}$ is given to player $i$. If some player $i$ is chosen to be monitored in some period $t$, the reports are examined at the end of $t$. Specifically, the mediator checks if the reports collected "match" the instruction given to $i$. The punishment phase begins for this component game if and only if there is a mismatch. The same review process is repeated at the end of every period in the cooperation phase. Given this $\tau$, the strategy profile $\sigma^{*}$ is specified as follows: Let $\mu_{i}^{*}$ be player $i$ 's reporting rule that reports his private signal truthfully, and $\lambda_{i}^{*}$ be the action rule that obeys the mediator's instruction. Let $\sigma_{i}^{*}$ be player $i$ 's honest and obedient strategy that plays the combination $c_{i}^{*}=\left(\lambda_{i}^{*}, \mu_{i}^{*}\right)$ for any pair of communication and private histories. It is clear that the strategy profile $\sigma^{*}$ is simple.

The mediation strategy $\tau$ is formally described as follows. Let $T$ be an integer and suppose that the sets of instructions and reports are given by $Q_{i}=A_{i} \cup\{\mathrm{NE}\}$ and $R_{i}=S_{i}$, respectively, where "NE" stands for (one-shot) "Nash equilibrium." Consider the following (partitioned) grim-trigger mediation strategy $\tau$ with the cooperation and punishment phases:

a) If the game is in the cooperation phase in some period $t$, choose the instructions for period $t$ as follows:

\footnotetext{
${ }^{12}$ The idea of a partitioned repeated game originates with Ellison (1994) and is used by Sekiguchi (1997).
} 
i) The mediator first determines whether or not any player will be monitored in period $t$. Let $m(t)=i$ if player $i$ is to be monitored, and $m(t)=\phi$ if no monitoring is to take place. The probability that any single player is monitored equals $\eta \in(0,1 / n)$. (Hence, the probability that monitoring takes place equals $n \eta$.)

ii) If monitoring is to take place in period $t$ (i.e., $m(t) \in I)$, the mediator chooses an action profile from $A$ according to the uniform probability distribution $\theta$ :

$$
\theta(a)=\frac{1}{|A|} \quad \text { for any } a \in A \text {. }
$$

On the other hand, if no monitoring is to take place in period $t$ (i.e, $m(t)=\phi$ ), then the mediator chooses an action profile from $A$ according to the probability distribution $\omega$ such that

$$
v_{i}^{*}=\sum_{a \in A} \omega(a) g_{i}(a) \quad \text { for every } i \in I \text {. }
$$

The monitoring decisions as well as the choice of action profiles are independent across periods. Whether monitoring is performed or not, if $a \in A$ is the chosen action profile, then player $i$ receives the instruction $q_{i}(t)=a_{i}$. It should be noted that such an instruction indicates neither the absence of the monitoring activity nor the identity of the monitored player (if any). ${ }^{13}$

b) If the game is in the punishment phase in period $t$, the instruction for period $t$ is "NE" for every player.

c) The transition of the phases in each component game is determined as follows: Given the instruction profile $q$ and the report profile $r$ in period $t$, let the random variable $\tilde{f}=1$ if

$$
\tilde{m}=i \text { and } \tilde{r}_{-i} \notin \Omega_{-i}\left(\tilde{q}_{i}\right) \text { for some } i \in I \text {, }
$$

and $\tilde{f}=0$ otherwise. In other words, $\tilde{f}=1$ indicates that some player $i$ is monitored, and that the report profile of others does not match the instruction given to $i$. Note that in terms of $a_{i}^{*}: S_{-i} \rightarrow A_{i}$ defined in Section $3, \tilde{r}_{-i} \notin \Omega_{-i}\left(\tilde{q}_{i}\right)$ is equivalent to

$$
\tilde{q}_{i} \neq a_{i}^{*}\left(\tilde{r}_{-i}\right)
$$

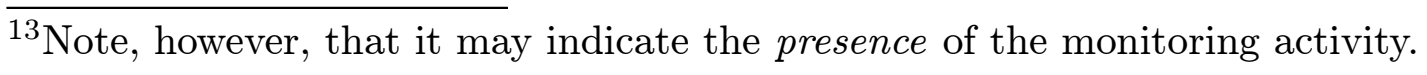


The cooperation phase continues in this component game if $\tilde{f}=0$, and the punishment phase begins otherwise. The same review process is repeated at the end of every period as long as the game is in the cooperation phase.

It will be shown below that $\tau$ coupled with the honest and obedient strategy profile $\sigma^{*}$ is a communication equilibrium, and that the equilibrium payoff is close to $v_{i}^{*}$ with an appropriate choice of $\epsilon, \delta$ and $T$. With slight abuse of notation, we use $P(\cdot \mid c)$ to denote the probability distribution of $(\tilde{m}, \tilde{f}, \tilde{q}, \tilde{s})$ under the profile $c$ in the cooperation phase of $\tau$. (Note that the dependence on $\tau$ is suppressed.)

The following lemma provides the key observation that the honest reporting rule $\mu_{i}^{*}$ is optimal combined with any action rule $\lambda_{i}$.

Lemma 1. Suppose that monitoring is jointly $\epsilon$-perfect and consistent. Then for any $j \in I$ and any pair $\left(\lambda_{j}, \mu_{j}\right)$ of action and reporting rules,

$$
P\left(\tilde{f}=0 \mid \lambda_{j}, \mu_{j}, c_{-j}^{*}\right) \leq P\left(\tilde{f}=0 \mid \lambda_{j}, \mu_{j}^{*}, c_{-j}^{*}\right)
$$

Proof: See the Appendix.

To see the logic behind Lemma 1 , suppose for simplicity that $n=2$. If player $j$ has received signal $s_{j}$ about player $i, j$ needs to choose his report $r_{j}$ so as to maximize the probability of the correct match $r_{j} \in \Omega_{j}\left(q_{i}\right)$ through the inference of the instruction $q_{i}$ given to player $i$. Given that the choice of an action profile by the mediator is uniform (conditional on monitoring being under way), congruency implies that $i$ has most likely been instructed to play $a_{i}^{*}\left(s_{j}\right)$. Since $s_{j} \in \Omega_{j}\left(a_{i}^{*}\left(s_{j}\right)\right)$, truth-telling is indeed optimal. When congruency fails as in Figure 1, $j$ 's incentive in reporting is affected by his own action $a_{j}$ because the most likely action of $i$ given $s_{j}=2$ changes with $a_{j}$. This implies that Lemma 1 would not hold.

We now examine each player's incentive to obey the instruction. First, let $v_{i}(\delta)$ denote player $i$ 's payoff from $\left(\sigma_{i}^{*}, \tau\right)$ in any component game. Since the payoff from every component game is the same, the average discounted payoff in the entire repeated game $V_{i}\left(\sigma^{*}, \tau, \delta\right)$ equals $v_{i}(\delta)$ as well. Given that the effective discount factor in each component game is given by $\delta^{T}, v_{i}(\delta)$ satisfies the following recursive equation:

$$
v_{i}(\delta)=\left(1-\delta^{T}\right) g_{i}^{\eta}+\delta^{T} P\left(\tilde{f}=0 \mid c^{*}\right) v_{i}(\delta),
$$


where $g_{i}^{\eta}=(1-n \eta) v_{i}^{*}+n \eta \sum_{a \in A} \theta(a) g_{i}(a)$. Let $\alpha=P\left(\tilde{f}=1 \mid c^{*}\right)$ be the (ex ante) probability that the report does not match the instruction when every player obeys the instruction and reports their signal truthfully. It follows from joint $\epsilon$-perfection that

$$
\alpha=\eta \sum_{i \in I} \sum_{a \in A} \theta(a) P\left(\tilde{s}_{-i} \notin \Omega_{-i}\left(a_{i}\right) \mid a\right) \leq n \eta \epsilon .
$$

Since $P\left(\tilde{f}=0 \mid c^{*}\right)=1-\alpha$, solving (4) for $v_{i}(\delta)$ yields

$$
v_{i}(\delta)=\frac{\left(1-\delta^{T}\right) g_{i}^{\eta}}{1-\delta^{T}(1-\alpha)} .
$$

Suppose next that player $i$ is given the instruction $\tilde{q}_{i}=a_{i}$ in the cooperation phase but deviates to $a_{i}^{\prime}\left(\neq a_{i}\right)$. Given consistencty, no combinatorial deviation in the action and reporting rules is profitable if no deviation in the action rule alone is profitable (Lemma 1 ). Let $\lambda_{i}$ be $i$ 's pure action rule such that $\lambda_{i}\left(a_{i}\right)=a_{i}^{\prime}$. Player $i$ 's expected continuation payoff from playing $c_{i}=\left(\lambda_{i}, \mu_{i}^{*}\right)$ is bounded from above by

$$
\left(1-\delta^{T}\right) \bar{g}_{i}+\delta^{T} P\left(\tilde{f}=0 \mid \tilde{q}_{i}=a_{i}, c_{i}, c_{-i}^{*}\right) v_{i}(\delta),
$$

where $\bar{g}_{i}=\max _{a \in A} g_{i}(a)$. On the other hand, when $i$ obeys the instruction, his payoff is bounded from below by

$$
\left(1-\delta^{T}\right) \underline{g}_{i}+\delta^{T} P\left(\tilde{f}=0 \mid \tilde{q}_{i}=a_{i}, c^{*}\right) v_{i}(\delta),
$$

where $\underline{g}_{i}=\min _{a \in A} g_{i}(a)$. Therefore, no one-step deviation is profitable if

$$
\delta^{T}\left\{P\left(\tilde{f}=1 \mid \tilde{q}_{i}=a_{i}, c_{i}, c_{-i}^{*}\right)-P\left(\tilde{f}=1 \mid \tilde{q}_{i}=a_{i}, c^{*}\right)\right\} v_{i}(\delta) \geq\left(1-\delta^{T}\right)\left(\bar{g}_{i}-\underline{g}_{i}\right) .
$$

In the Appendix, it will be shown that this inequality holds and that $v_{i}(\delta)$ is close to $v_{i}^{*}$ for a sufficiently large $\delta$ when $T$ is appropriately chosen.

Example 4: Suppose that the stage-game is the prisoners' dilemma with $A_{i}=\{C, D\}$, $S_{i}=\{0,1\}$, and the expected stage-payoff function $g_{i}: A \rightarrow \mathbf{R}$ depicted below $(x, y>0$, and $x-y<1)$.

$$
\begin{aligned}
& a_{2} \\
& \text { C D } \\
& \begin{array}{ccccc} 
& C & 1, & 1 & -y, 1+x \\
a_{1} & D & 1+x,-y & 0, & 0
\end{array}
\end{aligned}
$$

Figure 2 
The probability distribution $P$ is such that for some $\epsilon \in(0,1 / 2), P\left(\tilde{s}_{j}=0 \mid a_{j}, a_{i}=C\right) \geq$ $1-\epsilon$ and $P\left(s_{j}=1 \mid a_{j}, a_{i}=D\right) \geq 1-\epsilon$ for each $a_{j} \in A_{j}$. It follows that monitoring is $\epsilon$-perfect with $\Omega_{j}\left(a_{i}=C\right)=\{0\}$ and $\Omega_{j}\left(a_{i}=D\right)=\{1\}(i \neq j)$. Since $\epsilon<1 / 2$, consistency (congruency) holds by Proposition 2.

Consider now a communication equilibrium in which the efficient action profile $(C, C)$ is to be sustained (i.e., $\omega(C, C)=1$ ). We examine $i$ 's incentive to obey the instruction $q_{i}=C$. The instantaneous gain from the deviation to $a_{i}^{\prime}=D$ is given by

$$
\bar{g}_{i}(C)-\underline{g}_{i}(C)=(1+x) \frac{1-3 \eta / 2}{1-\eta}-\frac{1-(3+y) \eta / 2}{1-\eta}=\frac{x-(3 x-y) \eta / 2}{1-\eta} .
$$

Also, the difference in the (interim) probabilities of a mismatch in reporting (given $q_{i}=C$ ) when $i$ chooses $a_{i}^{\prime}=D$ and when he chooses $a_{i}=C$ satisfies $q-p \geq \frac{(1-2 \epsilon) \eta}{1-\eta}$. On the other hand, when the players are honest and obedient, the ex ante probability of a mismatch satisfies $\alpha \leq 2 \epsilon \eta$ by (5), and the ex ante stage-payoff equals $g_{i}^{\eta}=1-\eta+\frac{\eta}{2}(x-y)$. As seen in the proof of Theorem 1, there exists a communication equilibrium with payoff $v_{i}>1-\xi$ $(\xi>0)$ for a sufficiently large discount factor if

$$
\frac{\bar{g}_{i}(C)-\underline{g}_{i}(C)}{(q-p)\left(v_{i}^{*}-\xi\right)+\bar{g}_{i}(C)-\underline{g}_{i}(C)}<\frac{g_{i}^{\eta}-v_{i}^{*}+\xi}{g_{i}^{\eta}-(1-\alpha)\left(v_{i}^{*}-\xi\right)} .
$$

This is implied by

$$
\xi>\eta-\frac{\eta}{2}(x-y)+\frac{2 \epsilon}{1-2 \epsilon}\left\{x-\frac{\eta}{2}(3 x-y)\right\}
$$

Since $\eta>0$ can be taken arbitrarily small, we conclude that under $\epsilon$-perfection, there exists a communication equilibrium with payoff

$$
v_{i} \geq 1-\frac{2 \epsilon}{1-2 \epsilon} x
$$

provided that the players are sufficiently patient. When $x=1$, for example, $v_{i}=.75$ is sustained as an equilibrium payoff if $\epsilon<.1$, and $v_{i}=.95$ is sustained if $\epsilon<0.0238$. Note in particular that the specification of the joint distribution of $s=\left(s_{1}, s_{2}\right)$ is not important for this conclusion.

\section{Public vs. Mediated Communication}


In this section, we compare public and mediated communication in a simple twoperson game. We define a public communication game to be a communication game in which the mediation strategy $\tau$ is restricted so that instructions to all players are always the same: $\tilde{q}_{1}=\cdots=\tilde{q}_{n}$. A public equilibrium is a simple communication equilibrium of a public communication game. As mentioned in the Introduction, players' actions in public equilibrium are conditioned on the public reports of their private signals only. A public equilibrium is perfect if it induces a public equilibrium after every communication (public) history. Public communication under private monitoring is studied by Matsushima (1991), Kandori and Matsushima (1998), Compte (1998), and Aoyagi (2000), among others.

Consider now the following two-person normal-form game: The buyer (player 1) chooses whether to buy from the seller (player 2) or not. The seller, on the other hand, chooses either high or low effort, which stochastically determines the quality of the good (high or low). Formally, let $A_{1}=\{B, N\}$ and $A_{2}=\{H, L\}$ denote the sets of actions, and $S_{1}=\{l, h\}$ and $S_{2}=A_{1}$ denote the sets of signals. Player 1's action $B$ (resp. $N$ ) represents his choice to buy (resp. not to buy) from player 2, and 2's action $H$ (resp. $L$ ) represents his choice of high (resp. low) effort. Player 1's signals $l$ and $h$ correspond to "low" and "high" qualities, respectively. The probability distribution is such that $P\left(\tilde{s}_{2}=B \mid a_{1}=B, a_{2}\right)=P\left(\tilde{s}_{2}=N \mid a_{1}=N, a_{2}\right)=1$ for any $a_{2}$ so that player 2 perfectly monitors player 1 , while $P\left(\tilde{s}_{1}=l \mid a_{1}, a_{2}=L\right) \geq 1-\epsilon$ and $P\left(\tilde{s}_{1}=h \mid a_{1}, a_{2}=H\right) \geq 1-\epsilon$ for any $a_{1}$ so that player $1 \epsilon$-perfectly monitors player 2 regardless of his own action. ${ }^{14}$ Note that $s_{1}$ and $s_{2}$ are conditionally independent in the sense that $P\left(s_{1}, s_{2} \mid a\right)=P\left(s_{1} \mid a\right) P\left(s_{2} \mid a\right)$ for any $a \in A$. The stage-payoffs are such that $g_{1}(B, L)<g_{1}(N, L)=0 \leq g_{1}(N, H)<g_{1}(B, H)$ and $g_{2}(N, H) \leq g_{2}(N, L)=$ $0<g_{2}(B, H)<g_{2}(B, L)$. It follows that the profile $\left(a_{1}, a_{2}\right)=(N, L)$ is the one-shot Nash equilibrium, and that $\left(a_{1}, a_{2}\right)=(B, H)$ is efficient. Figure 3 depicts the set $V$ of feasible payoffs when $g_{1}(N, H)=g_{1}(N, L)$ and $g_{2}(N, H)=g_{2}(N, L)$. By Theorem 1 , any strictly positive payoff is approximated by a simple communication equilibrium payoff if $\epsilon$ is sufficiently small and $\delta$ is sufficiently large.

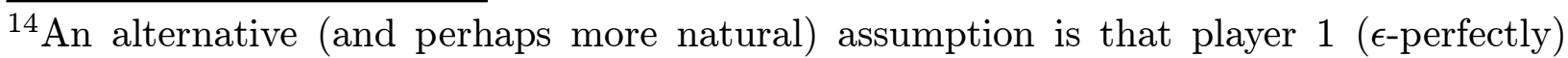
monitors 2's action only when he chooses to buy. While it violates the conditions of jointly $\epsilon$-perfect monitoring, it is not difficult to accommodate this alternative assumption into our theorem for this particular game.
} 
As seen in both Compte (1998) and Kandori and Matsushima (1994, 1998), the folk theorems based on public communication in general two-person games support only a subset of the individually rational payoffs. The problem is particularly serious in twoperson games. Specifically, Compte (1994) proves a theorem that demonstrates the limit of equilibrium payoffs based on public communication. His theorem applied to the above game shows that no perfect public equilibrium sustains an outcome close to the efficient outcome $\left(g_{1}(B, H), g_{2}(B, H)\right)$ regardless of the level of monitoring $\epsilon$. Formally, let

$$
W=\left\{\left(w_{1}, w_{2}\right): w_{1}=g_{1}(a) \text { and } w_{2}=\max _{a_{2}^{\prime} \in A_{2}} g_{2}\left(a_{1}, a_{2}^{\prime}\right) \text { for some } a=\left(a_{1}, a_{2}\right) \in A\right\}
$$

and

$$
\underline{V}^{2}=\left\{\left(v_{1}, v_{2}\right): v_{1} \leq w_{1} \text { and } v_{2} \geq w_{2} \text { for some } w \in \operatorname{co} W\right\}
$$

The set $\underline{V}^{2}$ is the shaded region in Figure 3. Note in particular that $\left(g_{1}(B, H), g_{2}(B, H)\right) \notin$ $\underline{V^{2}}$.

Proposition 3. (Compte (1998)) Suppose that $0<P\left(\tilde{s}_{1}=l \mid a_{1}, H\right)<P\left(\tilde{s}_{1}=l \mid\right.$ $\left.a_{1}, L\right)<1$ for $a_{1} \in A_{1}$. Then every perfect public equilibrium payoff vector belongs to the set $\underline{V}^{2}$ for any $\epsilon>0$ and $\delta$.

The proof is included in the Appendix for the reader's convenience. ${ }^{15}$

One interesting application of the current analysis concerns the problem of "coordinated boycott" as studied by Klein and Leffler (1980). When a single seller plays this game against many buyers, Klein and Leffler (1980) argue that the seller's incentive to exert high effort can be maintained by the threat of coordinated boycott by the buyers. The seller is prevented from cheating any single buyer since information about the quality of each individual good is shared by all the buyers. Implicit in their logic, therefore, is the assumption that each buyer willingly reveals the quality of their good to others including the seller. The above discussion suggests, however, that public communication does not yield the desired conclusion.

The key to the success of mediated communication is the inducement of truth-telling by the buyer through the seller's choice of low effort with positive probability. In other ${ }^{15}$ Compte's (1998) result applies to any two-person games with conditionally independent
signals. 
words, the buyer has no incentive to report the low quality and give up all future surplus if he believes that the seller chooses high effort with probability one. As mentioned in the Introduction, this is the essence of the observation made by Bhaskar and van Damme (1997). A related observation can also be found in the analysis of a reputation game between a seller and buyers by Mailath and Samuelson (1998), who argue that the seller's incentive to maintain his reputation is strongest when there is a substantial probability that he is the "inept" type who never exerts high effort.

\section{Appendix}

Proof of Proposition 1: For every $i \in I$, set

$$
a_{i}^{*}\left(s_{-i}\right)=s_{i-1}^{i}
$$

(For $i=1$, let $s_{0}^{1}=s_{n}^{1}$.) Take any $i \in I$ and $j \neq i$. If $j \neq i-1$, then (2) holds with equality since $a_{i}^{*}\left(s_{j}, s_{-i-j}\right)=a_{i}^{*}\left(r_{j}, s_{-i-j}\right)$ for any $s_{j}, r_{j} \in S_{j}$ by the above specification of $a_{i}^{*}$. Suppose that $j=i-1$. If $r_{j}^{i}=s_{j}^{i}$, then (2) holds with equality again. Suppose hence that $r_{j}^{i} \neq s_{j}^{i}$. For any $a_{j} \in A_{j}$, we have

$$
\begin{aligned}
& \sum_{a_{-i-j}} \sum_{s_{-i-j}} P\left(s_{j}, s_{-i-j} \mid a_{i}=a_{i}^{*}\left(s_{j}, s_{-i-j}\right), a_{j}, a_{-i-j}\right) \\
& \geq P\left(s_{j}, \tilde{s}_{k}=\left(a_{j}, s_{j}^{-j-k}\right) \text { for every } k \neq i, j \mid a_{i}=s_{j}^{i}, a_{j}, a_{-i-j}=s_{j}^{-i-j}\right) \\
& \geq 1-\epsilon,
\end{aligned}
$$

and

$$
\begin{aligned}
& \sum_{a_{-i-j}} \sum_{s_{-i-j}} P\left(s_{j}, s_{-i-j} \mid a_{i}=a_{i}^{*}\left(r_{j}, s_{-i-j}\right), a_{j}, a_{-i-j}\right) \\
& =\sum_{a_{-i-j}} \sum_{s_{-i-j}} P\left(s_{j}, s_{-i-j} \mid a_{i}=r_{j}^{i}, a_{j}, a_{-i-j}\right) \\
& \leq\left|A_{-i-j}\right|\left|S_{-i-j}\right| \epsilon .
\end{aligned}
$$

Therefore, (2) holds if $\epsilon \leq\left(1+\left|A_{-i-j}\right|\left|S_{-i-j}\right|\right)^{-1} \cdot / /$

Proof of Proposition 2: Let $P$ be jointly $\epsilon$-perfect with partitions $\left\{\Omega_{-i}\right\}_{i \in I}$. Take any $s_{-i} \in S_{-i}$ and $a_{-i} \in A_{-i}$, and suppose that

$$
\max _{a_{i} \in A_{i}} P\left(s_{-i} \mid a_{i}, a_{-i}\right) \geq \epsilon
$$


If $P\left(s_{-i} \mid a_{i}=a_{i}^{*}\left(s_{-i}\right), a_{-i}\right)<\max _{a_{i} \in A_{i}} P\left(s_{-i} \mid a_{i}, a_{-i}\right)$, then there exists $a_{i} \neq a_{i}^{*}\left(s_{-i}\right)$ such that $P\left(s_{-i} \mid a_{i}, a_{-i}\right) \geq \epsilon$. Since $s_{-i} \in \Omega_{-i}\left(a_{i}^{*}\left(s_{-i}\right)\right)$, it follows that $P\left(\tilde{s}_{-i} \in\right.$ $\left.\Omega_{-i}\left(a_{i}^{*}\left(s_{-i}\right)\right) \mid a_{i}, a_{-i}\right) \geq \epsilon$. Since $\Omega_{-i}\left(a_{i}\right) \cap \Omega_{-i}\left(a_{i}^{*}\left(s_{-i}\right)\right)=\phi$, this further implies $P\left(\tilde{s}_{-i} \in \Omega_{-i}\left(a_{i}\right) \mid a_{i}, a_{-i}\right)<1-\epsilon$, contradicting the joint $\epsilon$-perfection of $P$. //

Proof of Lemma 1: Recall that $a_{i}^{*}\left(s_{-i}\right)$ is defined to be the unique $a_{i} \in A_{i}$ such that $s_{-i} \in \Omega_{-i}\left(a_{i}\right)$. Consider the event $\tilde{f}=0$ that no mismatch in reporting is registered. Fix any action rule $\lambda_{j}$ of player $j$. When player $j$ uses the honest reporting rule $\mu_{j}^{*}$ along with $\lambda_{j}$, the probability of the above event is given by

$$
\begin{aligned}
P\left(\tilde{f}=0 \mid \lambda_{j}, \mu_{j}^{*}, c_{-j}^{*}\right) & =P(\tilde{m}=\phi) \\
& +\sum_{\substack{a_{-j} \in A_{-j} \\
s_{-j} \in S_{-j}\\
}} P(\tilde{m}=j) P\left(s_{-j} \mid \lambda_{j}\left(a_{j}^{*}\left(s_{-j}\right)\right), a_{-j}\right) \\
& \left.+\sum_{-j \neq j} \sum_{\substack{a_{-i} \in A_{-i} \\
s_{-i} \in S_{-i}}} P(\tilde{m}=i) P\left(\tilde{q}_{-i}=a_{-i}^{*}, \tilde{q}_{i}=a_{-j}^{*}\right) \mid \tilde{m}=j\right) \\
& \quad \times P\left(s_{-i} \mid a_{i}=a_{i}^{*}\left(s_{-i}\right), \lambda_{j}\left(a_{j}\right), a_{-i-j}\right),
\end{aligned}
$$

where the first term on the right-hand side corresponds to the event that no player is monitored, the second to the event that $j$ himself is monitored, and the third to the event that some other player is monitored. Suppose now that player $j$ uses an alternative reporting rule $\mu_{j}$. In this case, reports about $i$ are "correct" if and only if $q_{i}=a_{i}^{*}\left(\mu_{j}\left(s_{j}\right), s_{-i-j}\right)$ provided that the signal profile is $\left(s_{j}, s_{-i-j}\right)$. The probability of the event $\tilde{f}=0$ is hence given by

$$
\begin{aligned}
& P\left(\tilde{f}=0 \mid \lambda_{j}, \mu_{j}, c_{-j}^{*}\right)=P(\tilde{m}=\phi) \\
& +\sum_{\substack{a_{-j} \in A_{-j} \\
s_{-j} \in S_{-j}}} P(\tilde{m}=j) P\left(\tilde{q}_{-j}=a_{-j}, \tilde{q}_{j}=a_{j}^{*}\left(s_{-j}\right) \mid \tilde{m}=j\right) \\
& \times P\left(s_{-j} \mid \lambda_{j}\left(a_{j}^{*}\left(s_{-j}\right)\right), a_{-j}\right) \\
& +\sum_{i \neq j} \sum_{\substack{a-i \\
s_{-i} \in S_{-i} \\
s_{-i}}} P(\tilde{m}=i) P\left(\tilde{q}_{-i}=a_{-i}, \tilde{q}_{i}=a_{i}^{*}\left(\mu_{j}\left(s_{j}\right), s_{-i-j}\right) \mid \tilde{m}=i\right) \\
& \times P\left(s_{-i} \mid a_{i}=a_{i}^{*}\left(\mu_{j}\left(s_{j}\right), s_{-i-j}\right), \lambda_{j}\left(a_{j}\right), a_{-i-j}\right) .
\end{aligned}
$$


Since $P(\tilde{m}=i)=\eta$ and $P(q \mid \tilde{m}=i)=1 /|A|$ for any $i \in I$ and $q \in A$, we have

$$
\begin{aligned}
& P\left(\tilde{f}=0 \mid \lambda_{j}, \mu_{j}^{*}, c_{-j}^{*}\right)-P\left(\tilde{f}=0 \mid \lambda_{j}, \mu_{j}, c_{-j}^{*}\right) \\
& =\frac{\eta}{|A|} \sum_{i \neq j} \sum_{\substack{a-i \in A_{-i} \\
s_{-i} \in S_{-i}}}\left\{P\left(s_{-i} \mid a_{i}=a_{i}^{*}\left(s_{-i}\right), \lambda_{j}\left(a_{j}\right), a_{-i-j}\right)\right. \\
& \left.-P\left(s_{-i} \mid a_{i}=a_{i}^{*}\left(\mu_{j}\left(s_{j}\right), s_{-i-j}\right), \lambda_{j}\left(a_{j}\right), a_{-i-j}\right)\right\} \\
& =\frac{\eta}{|A|} \sum_{i \neq j} \sum_{\substack{a_{j} \in A_{j} \\
s_{j} \in S_{j}}}\left[\sum _ { \substack { a _ { - i - j } \in A _ { - i - j } \\
s _ { - i - j } \in S _ { - i - j } } } \left\{P\left(s_{-i} \mid a_{i}=a_{i}^{*}\left(s_{-i}\right), \lambda_{j}\left(a_{j}\right), a_{-i-j}\right)\right.\right. \\
& \left.\left.-P\left(s_{-i} \mid a_{i}=a_{i}^{*}\left(\mu_{j}\left(s_{j}\right), s_{-i-j}\right), \lambda_{j}\left(a_{j}\right), a_{-i-j}\right)\right\}\right] .
\end{aligned}
$$

The desired conclusion follows since consistency implies that the quantity in the square brackets on the right-hand side is greater than or equal to zero. //

Proof of Theorem 1: Let

$$
p=P\left(\tilde{f}=1 \mid \tilde{q}_{i}=a_{i}, c^{*}\right) \quad \text { and } \quad q=P\left(\tilde{f}=1 \mid \tilde{q}_{i}=a_{i}, c_{i}, c_{-i}^{*}\right)
$$

denote the (interim) probabilities of a mismatch when player $i$ obeys the instruction $\tilde{q}_{i}=$ $a_{i}$, and when he disobeys and plays $\lambda_{i}\left(a_{i}\right)=a_{i}^{\prime}$ instead, respectively. Joint $\epsilon$-perfection implies that

$$
\begin{aligned}
p & =\sum_{j \in I} \sum_{a_{-i} \in A_{-i}} P\left(\tilde{s}_{-j} \notin \Omega_{-j}\left(a_{j}\right) \mid a_{i}, a_{-i}\right) P\left(\tilde{q}_{-i}=a_{-i}, \tilde{m}=j \mid \tilde{q}_{i}=a_{i}\right) \\
& \leq \epsilon .
\end{aligned}
$$

On the other hand, $q$ is given by

$$
\begin{aligned}
q & =\sum_{a_{-i} \in A_{-i}} P\left(\tilde{s}_{-i} \notin \Omega_{-i}\left(a_{i}\right) \mid a_{i}^{\prime}, a_{-i}\right) P\left(\tilde{q}_{-i}=a_{-i}, \tilde{m}=i \mid \tilde{q}_{i}=a_{i}\right) \\
& +\sum_{j \neq i} \sum_{a_{-i} \in A_{-i}} P\left(\tilde{s}_{-j} \notin \Omega_{-j}\left(a_{j}\right) \mid a_{i}^{\prime}, a_{-i}\right) P\left(\tilde{q}_{-i}=a_{-i}, \tilde{m}=j \mid \tilde{q}_{i}=a_{i}\right),
\end{aligned}
$$

where the first term on the right-hand side corresponds to the event that player $i$ himself is monitored, while the second corresponds to the event that some other player is monitored. 
Since for any $j \in I$,

$$
\begin{aligned}
& P\left(\tilde{q}_{-i}=a_{-i}, \tilde{m}=j \mid \tilde{q}_{i}=a_{i}\right) \\
& =\frac{P(\tilde{q}=a \mid \tilde{m}=j) P(\tilde{m}=j)}{\sum_{j^{\prime} \in I} P\left(\tilde{q}_{i}=a_{i} \mid \tilde{m}=j^{\prime}\right) P\left(\tilde{m}=j^{\prime}\right)+P\left(\tilde{q}_{i}=a_{i} \mid \tilde{m}=\phi\right) P(\tilde{m}=\phi)} \\
& \in\left[\frac{\eta}{|A|}, \frac{1}{\left|A_{-i}\right| n}\right],
\end{aligned}
$$

it follows from joint $\epsilon$-perfection that

$$
q \geq(1-\epsilon) \frac{\eta}{\left|A_{i}\right|}
$$

For any $\xi>0$, take $\eta$ small enough so that $\left|g_{i}^{\eta}-v_{i}^{*}\right|<\xi / 2$ for every $i \in I$. It then follows from (6) that $v_{i}(\delta) \leq g_{i}^{\eta}<v_{i}^{*}+\xi$ for every $i \in I$. Take $\epsilon>0$ small enough so that Lemma 1 holds and that

$$
\max _{i \in I} \frac{\bar{g}_{i}-\underline{g}_{i}}{(q-p)\left(v_{i}^{*}-\xi\right)+\bar{g}_{i}-\underline{g}_{i}}<\min _{i \in I} \frac{g_{i}^{\eta}-v_{i}^{*}+\xi}{g_{i}^{\eta}-(1-\alpha)\left(v_{i}^{*}-\xi\right)} .
$$

This is possible since as $\epsilon \rightarrow 0, \alpha \rightarrow 0$ by (5), $p \rightarrow 0$ by (a1), and $q>0$ is bounded away from zero by (a2). We now take $\underline{\delta}<1$ large enough so that for any $\delta>\underline{\delta}$, there exists an integer $T$ such that

$$
\max _{i \in I} \frac{\bar{g}_{i}-\underline{g}_{i}}{(q-p)\left(v_{i}^{*}-\xi\right)+\bar{g}_{i}-\underline{g}_{i}} \leq \delta^{T}<\min _{i \in I} \frac{g_{i}^{\eta}-v_{i}^{*}+\xi}{g_{i}^{\eta}-(1-\alpha)\left(v_{i}^{*}-\xi\right)} .
$$

The right inequality of (a3) and (6) together imply that $v_{i}(\delta)>v_{i}^{*}-\xi$. On the other hand, the incentive constraint (9) follows from the left inequality of (a3). This completes the proof of the theorem. //

Proof of Proposition 3: Let

$$
V^{p}=\left\{v=\left(v_{1}, v_{2}\right): v \text { is a perfect public equilibrium payoff vector }\right\}
$$

Take any perfect public equilibrium and let $v=\left(v_{1}, v_{2}\right)$ be the corresponding payoff vector. By the standard argument, there exist an action profile $a \in A$, a reporting rule $\mu_{i}$ : $A_{i} \times S_{i} \rightarrow R_{i}$, and the continuation payoff function $w_{i}: R \rightarrow V^{p}$ such that

$$
v_{i}=(1-\delta) g_{i}(a)+\delta \sum_{r \in R} P(r \mid a, \mu) w_{i}(r)
$$


and

$$
v_{i} \geq(1-\delta) g_{i}\left(a_{i}^{\prime}, a_{j}\right)+\delta \sum_{r \in R} P\left(r \mid a_{i}^{\prime}, a_{j}, \mu_{i}^{\prime}, \mu_{j}\right) w_{i}(r) \quad \text { for any }\left(a_{i}^{\prime}, \mu_{i}^{\prime}\right)(j \neq i)
$$

Take any $i \in I$ and $r_{i} \in R_{i}$, and consider $i$ 's deviation to the "constant" reporting rule $\mu_{i}^{\prime}$ such that $\mu_{i}^{\prime}\left(a_{i}, \cdot\right) \equiv r_{i}$. We have by $($ a5 $)$,

$$
v_{i} \geq(1-\delta) g_{i}(a)+\delta \sum_{r_{j} \in R_{j}} P\left(r_{j} \mid a, \mu_{j}\right) w_{i}\left(r_{i}, r_{j}\right)
$$

Since (a4) under conditional independence can be written as

$$
v_{i}=\sum_{r_{i} \in R_{i}} P\left(r_{i} \mid a, \mu_{i}\right)\left\{(1-\delta) g_{i}(a)+\delta \sum_{r_{j} \in R_{j}} P\left(r_{j} \mid a, \mu_{j}\right) w_{i}\left(r_{i}, r_{j}\right)\right\}
$$

it follows from (a6) that for every $r_{i} \in R_{i}$ with $P\left(r_{i} \mid a, \mu_{i}\right)>0$,

$$
v_{i}=(1-\delta) g_{i}(a)+\delta \sum_{r_{j} \in R_{j}} P\left(r_{j} \mid a, \mu_{j}\right) w_{i}\left(r_{i}, r_{j}\right)
$$

This further implies that if $P\left(r_{i} \mid a, \mu_{i}\right)>0$ and $P\left(r_{i}^{\prime} \mid a, \mu_{i}\right)>0$, then

$$
\sum_{r_{j} \in R_{j}} P\left(r_{j} \mid a, \mu_{j}\right) w_{i}\left(r_{i}, r_{j}\right)=\sum_{r_{j} \in R_{j}} P\left(r_{j} \mid a, \mu_{j}\right) w_{i}\left(r_{i}^{\prime}, r_{j}\right)
$$

Let $\bar{a}_{2} \in \arg \max _{a_{2}^{\prime} \in A_{2}} g_{2}\left(a_{1}, a_{2}^{\prime}\right)$. Since $P\left(r_{1} \mid a, \mu_{1}\right)>0$ if and only if $P\left(r_{1} \mid a_{1}, \bar{a}_{2}, \mu_{1}\right)>$ 0 for any $\left(a, \mu_{1}\right)$ by assumption, it follows from the above observation that

$$
v_{1}=(1-\delta) g_{1}(a)+\delta \sum_{r_{1} \in R_{1}} P\left(r_{1} \mid a_{1}, \bar{a}_{2}, \mu_{1}\right) \sum_{r_{2} \in R_{2}} P\left(r_{2} \mid a, \mu_{2}\right) w_{1}\left(r_{1}, r_{2}\right)
$$

On the other hand, consider player 2's deviation to $\left(\bar{a}_{2}, \bar{\mu}_{2}\right)$ such that $P\left(r_{2} \mid a_{1}, \bar{a}_{2}, \bar{\mu}_{2}\right)=$ $P\left(r_{2} \mid a, \mu_{2}\right)$ for every $r_{2}$. We have by (a5),

$$
v_{2} \geq(1-\delta) g_{2}\left(a_{1}, \bar{a}_{2}\right)+\delta \sum_{r_{1} \in R_{1}} P\left(r_{1} \mid a_{1}, \bar{a}_{2}, \mu_{1}\right) \sum_{r_{2} \in R_{2}} P\left(r_{2} \mid a, \mu_{2}\right) w_{2}\left(r_{1}, r_{2}\right) .
$$

It follows from (a7) and (a8) that for $\nu=\left(\nu_{1}, \nu_{2}\right)$ with $\nu_{1} \geq 0$ and $\nu_{2} \leq 0$,

$$
\begin{aligned}
\nu \cdot v & \leq(1-\delta) \nu \cdot\left(g_{1}(a), g_{2}\left(a_{1}, \bar{a}_{2}\right)\right) \\
& +\delta \sum_{r_{1} \in R_{1}} P\left(r_{1} \mid a_{1}, \bar{a}_{2}, \mu_{1}\right) \sum_{r_{2} \in R_{2}} P\left(r_{2} \mid a, \mu_{2}\right) \nu \cdot w\left(r_{1}, r_{2}\right),
\end{aligned}
$$


where $\cdot$ denotes the inner product. If we let $k(\nu, W)=\sup _{w \in \operatorname{co} W} \nu \cdot w$, then the above implies that for any $v=\left(v_{1}, v_{2}\right) \in V^{p}$,

$$
\nu \cdot v \leq(1-\delta) k\left(\nu, \underline{V}^{2}\right)+\delta k\left(\nu, V^{p}\right)
$$

Taking the supremum over $v \in V^{p}$ on the left-hand side, we obtain $k\left(\nu, V^{p}\right) \leq k\left(\nu, \underline{V}^{2}\right)$. Since this is true for any $\nu$ with $\nu_{1} \geq 0$ and $\nu_{2} \leq 0$, and since $\underline{V}^{2}$ is unbounded in the direction $-\nu$, we conclude that $V^{p} \subset \underline{V}^{2}$. //

\section{References}

Aoyagi, M. (2000), "Collusion in dynamic Bertrand oligopoly with correlated private signals and communication," mimeo.

Ben-Porath, E. and M. Kahneman (1996), "Communication in repeated games with private monitoring," Journal of Economic Theory, 70, 281-297.

Bhaskar, V. and E. Van Damme (1997), "Moral hazard and private monitoring," mimeo.

Compte, O. (1994), "Private observations, communication and coordination in repeated games," Ph.D. dissertation, Stanford University.

Compte, O. (1998), "Communication in repeated games with imperfect private monitoring," Econometrica, 66, 597-626.

Doyle, M. P. and C. M. Snyder (1999), "Information sharing and competition in the motor vehicle industry," Journal of Political Economy, 107, 1326-64.

Ellison, G. (1994), "Cooperation in the prisoners' dilemma with anonymous random matching," Review of Economic Studies, 61, 567-588.

Ely, J. and J. Valimaki (2000), "A robust folk theorem for the prisoners' dilemma," mimeo.

Fletcher, C. (1997), Appraisal - Routes to Improved Performance, 2nd Ed., London: Institute of Personnel Development.

Forges, F. (1986), "An approach to communication equilibria," Econometrica, 54, 13751385 .

Genesove, D. and W. P. Mullin (2001), "Rules, communication, and collusion: narrative evidence from the Sugar Institute case," American Economic Review, , 379-398.

Kandori, M. and H. Matsushima (1994), "Private observation, communication and collusion," Discussion Paper 94-F-33, Faculty of Economics, University of Tokyo. 
Kandori, M. and H. Matsushima (1998), "Private observation, communication and collusion," Econometrica, 66, 627-652.

Mailath, G. and S. Morris (1999), "Repeated games with almost-public monitoring," mimeo.

Mailath, G. and L. Samuelson (1998), "Your reputation is who you are not, not who you would like to be," mimeo.

Matsushima, H. (1990), "On the theory of repeated games with private information. Part II: Revelation through communication," Economics Letters, 35, 257-261.

Mitusch, K. and R. Strausz (2000), "Mediation in situations of conflict," mimeo.

Myerson, R. (1986), "Multistage games with communication," Econometrica, 54, 323-358.

Obara, I. (2000) "The repeated prisoners' dilemma with private monitoring: an $N$-player case," mimeo.

Piccione, M. (1999), "The repeated prisoners' dilemma with imperfect private monitoring," mimeo.

Scherer, F. M. (1980), Industrial Market Structure and Economic Performance, 2nd Ed., Boston: Houghton Mifflin.

Sekiguchi, T. (1997), "Efficiency in repeated prisoners' dilemma with private monitoring," Journal of Economic Theory, 76, 345-361.

Sekiguchi, T. (1998), "Robustness of efficient equilibria in repeated games with imperfect private monitoring," mimeo.

Stigler, G. (1964), "A theory of oligopoly," Journal of Political Economy, 72, 44-61. 


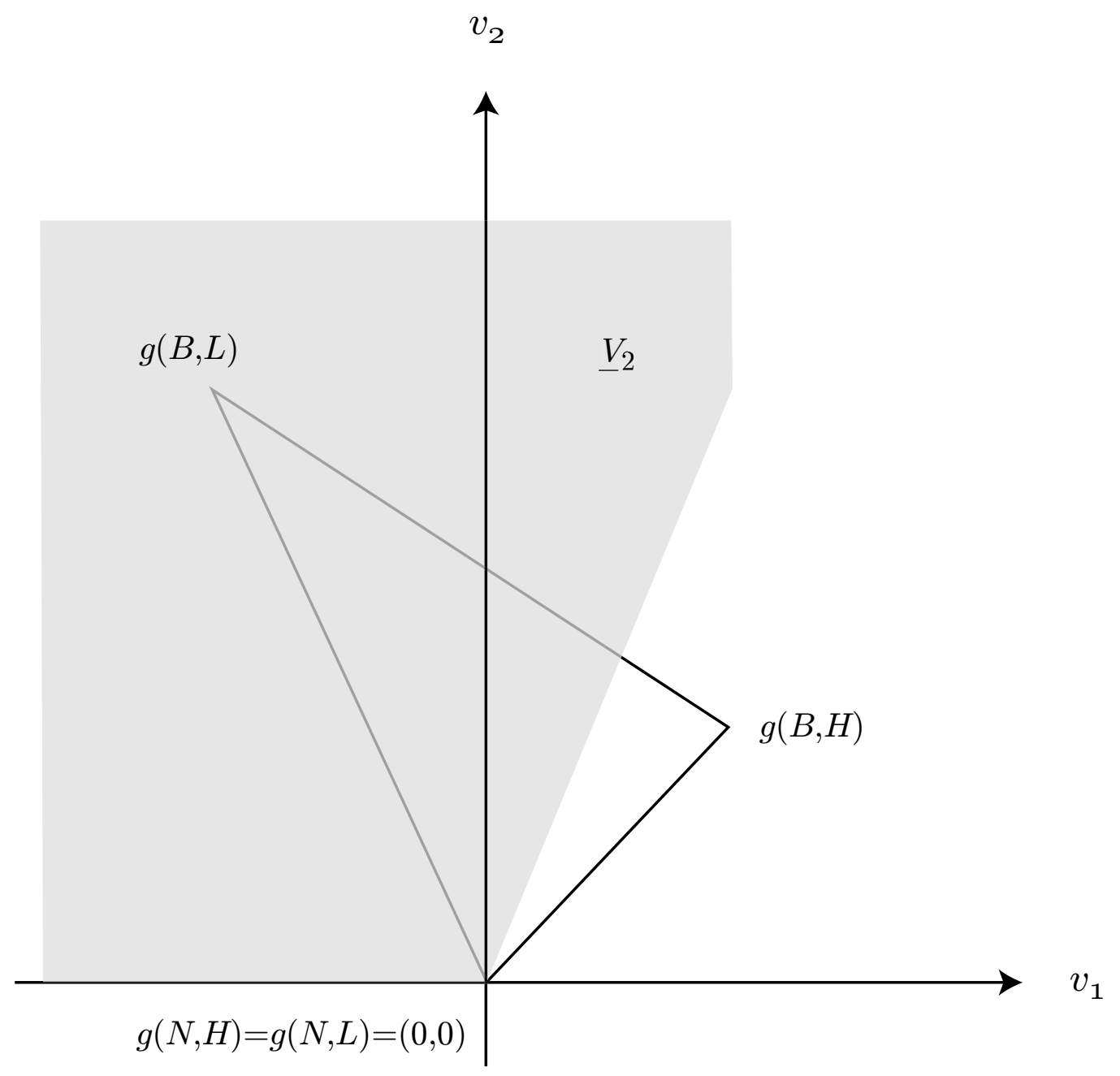

Figure 3 